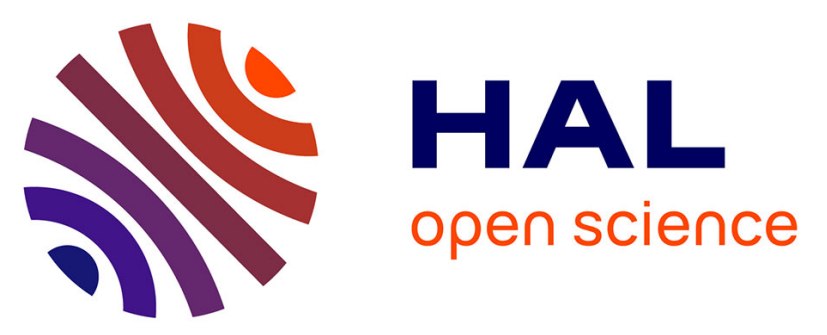

\title{
CORRELATION ENTRE LES DISLOCATIONS, LES CONCENTRATIONS EN CARBONE ET LES LONGUEURS DE DIFFUSION DANS DU SILICIUM POLYCRISTALLIN C.G.E
}

\author{
H. Amzil, L. Ammor, E. Psaila, M. Zehaf, G. Mathian, S. Martinuzzi, J. \\ Crest, J. Oualid, B. Pichaud, F. Minari
}

\section{To cite this version:}

H. Amzil, L. Ammor, E. Psaila, M. Zehaf, G. Mathian, et al.. CORRELATION ENTRE LES DISLOCATIONS, LES CONCENTRATIONS EN CARBONE ET LES LONGUEURS DE DIFFUSION DANS DU SILICIUM POLYCRISTALLIN C.G.E. Journal de Physique Colloques, 1983, 44 (C4), pp.C4-415-C4-422. 10.1051/jphyscol:1983449 . jpa-00223069

\section{HAL Id: jpa-00223069 https://hal.science/jpa-00223069}

Submitted on 1 Jan 1983

HAL is a multi-disciplinary open access archive for the deposit and dissemination of scientific research documents, whether they are published or not. The documents may come from teaching and research institutions in France or abroad, or from public or private research centers.
L'archive ouverte pluridisciplinaire HAL, est destinée au dépôt et à la diffusion de documents scientifiques de niveau recherche, publiés ou non, émanant des établissements d'enseignement et de recherche français ou étrangers, des laboratoires publics ou privés. 


\title{
CORRELATION ENTRE LES DISLOCATIONS, LES CONCENTRATIONS EN CARBONE ET LES LONGUEURS DE DIFFUSION DANS DU SILICIUM POLYCRISTALLIN C,G,E
}

\author{
H. Amzil, L. Ammor, E. Psaila, M. Zehaf, G. Mathian, S. Martinuzzi, \\ J.P. Crest ${ }^{*}$, J. Oualid*, B. Pichaud ${ }^{* *}$ et F. Minari* \\ Laboratoire de Photoélectricité des Semiconducteurs, \\ * Laboratoire des Matériaux et Composants à Semiconducteurs, \\ ** Laboratoire de Physique Cristaliine, \\ Université d'Aix-Marseizle III, Centre St Jêrôme, me H. Poincaré, \\ 13397 Marseizle Cedex, France
}

Rēsumé - Le silicium polycristallin étudiê, présente des dëfauts cristallographiques (joints de grains, dislocations...) qui ai tèrent ses propriétés êlectroniques. La répartition de ces défauts ètant inhomogène une êtude statistique a été faite sur des minidiodes mesa $\mathrm{N}^{+}-\mathrm{P}$ présentant approximativement la même longueur totale de joints de grains $L j$ pour mettre en évidence l'influence des dislocations. Les dislocations sont révélées par attaque chimique sélective ce qui conduit à des densités de dislocations minorées, variant entre $10^{3}$ et $10^{6} \mathrm{~cm}^{-3}$. Les évolutions des densités de dislocations observées en comptant les figures d'attaque sont confirmées par topographie $\mathrm{X}$.

Les longueurs de diffusion $L_{n}$ et les photocourants de court circuit $I_{c c}$ des diodes diminuent quand la densité de dislocations $N_{\text {dis }}$ augmente. Parallèlement $L_{n}$ et $I_{C c}$ diminuent quand la concentration en carbone [C] varie de

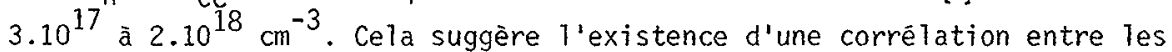
fortes densités de dislocations observées et les concentrations en carbone élevées mesurées par analyse infrarouge. En particuljer $\mathrm{N}_{\text {dis }}$ augmente rapidement quand $[C]$ dépasse la limite de solubilité.

Abstract - The polysilicon studied contains many cristallographic defects (grains boundaries, dislocations...) which degrade the electronic properties. As these defects are randomly distributed, the study of their detrimental effects needs the investigation of a large quantity of mesa diode $\mathrm{NPP}$ presenting approximately the same total length of grains boundaries $\mathrm{LJ}$ in order to obtain the influence of the dislocations. The dislocations densities. $N_{\text {dis }}$ were evaluated by counting etch pits revealed by chemical etching. The evolution of $N_{d i s}$ were confirmed by $X$ topography.

The photocurrents $I_{C c}$ of the diodes and the diffusion length $L_{n}$ decreased when $N_{d i s}$ increased. I $c c$ and $L_{n}$ decreased also markedly when the carbon concentration (determined by I.R. analysis) in the silicon varied from $3.10^{17}$ to $2.10^{18} \mathrm{~cm}^{-3}$. These results suggested that dislocations and excess carbon are correlated.

\section{I - INTRODUCTION}

Le silicium polycristallin est un matériau semi-conducteur susceptible d'être utilise dans la fabrication de composants électroniques et de photopiles solaires, sous forme de couches minces ou de plaquettes "semicristallines" découpées dans des lingots obtenus par croissance unidirectionnelle.

Les différents siliciums polycristallins (couches, rubans, plaquettes...) sont gênéralement préparés par des méthodes rapides afin d'abaisser le coút de fabrication. Il $s^{1}$ en suit que ces matériaux sont affectés par de nombreux défauts cristallographiques comme les joints de grains, les sous joints, les dislocations... Lorsque les densités de défauts intragrains sont faibles, et que la taille moyanne des grains dēpasse 
$500 \mu \mathrm{m}$, les joints de grains dégradent les propriétés électroniques (longueur de diffusion $L$ - durēe de vie $\tau$ ) et photovoltaĩques (photocourant de court-circuit I $c c$, tension de circuit ouvert $\left.V_{\text {co }} \ldots\right) / 1 /$.

Si les densitês de défauts intragrains sont élevêes, au contraire, l'infiuence des joints de grains est peu marquée. Les défauts intragrains les plus actifs et les plus facilement dénombrables sont les dislocations $/ 2,3 /$ (les impuretés recombinantes sont gënēralement en quantités trẽs faibles dans les polycristaux réalisés à partir de silicium de qualité électronique).

Les procédés d'êlaboration rapide de ces matériaux utilisent souvent un contact direct entre un creuse' ou un contcur en graphite et le silicium fondu ce qui entraine inévitablement la dissolution de carbone dans le silicium en quantitê plus ou moins importante suivant le procédé. Cela peut se traduire par une dissolution à peu près homogène sans conséquence sur les propriétés électroniques du matériau, ou par une sursaturation si la limite de solubilité est dépassée, ou encore dans les cas extrêmes par une précipitation avec formation de Sic qui créerait des dislocations $/ 4,5 /$.

Dans ce qui suit, nous mettons en évidence l'influence des dislocations sur la longueur de diffusion des porteurs minoritaires $L_{n}$ dans des plaquettes de silicium polycristallin de type $P$ dont la teneur en carbone varie entre $3.10^{17}$ et $2.10^{18} \mathrm{~cm}^{-3}$, et comme $L_{n}$ varie avec la concentration en carbone, nous nous efforçons d'établir une corrélation entre les densités de dislocations et les concentrations en carbone qui approchent ou dépassent la limite de solubilité.

\section{II - TECHNIQUES EXPERIMENTALES}

Le silicium polycristallin étudié est prēparé aux Laboratoires de Marcoussis CGE /6/ par une variante accélērée du procédé Bridgmann sans germe dans des creusets en graphite nus (lingot 121) ou partiellement revêtus d'une céramique pouvant isoler le graphite duisilicium en fusion (lingot 123). Cette croissance unidirectionnelle est faite sous vide. Les lingots de silicium de type $P$ (dopage au bore) sont découpés en plaquettes de $300 \mathrm{um}$ d'épaisseur qui sont transformées en photopiles par Photowatt S.A. après une brève diffusion de phosphore (Ih à $850^{\circ} \mathrm{C}$ ) destinèe à créer une jonction $\mathbb{N}^{+}-\mathrm{P}$ peu profonde. Les défauts étant répartis de façon très inhomogẽne dans Tes plaquettes, une étude statistique portant sur un grand nombre d'échantilions a été effectuée. Les plaquettes sont découpēes en ëchantillons de $2 \times 1 \mathrm{~cm}$ sur lesquelles 12 minidiodes mesa de $1,5 \times 1,5 \mathrm{~mm}$ sont révétées par photolithographie. Les méthodes de mesure des longueurs de diffusion à partir de la rêponse spectrale dans le proche infrarouge ont déjà étê décrites $/ 1 /$. Les dislocations sont révélées à 1 'emplacement des diodes mesas étudiées à 7 'aide du réactif de Sirtl qui développe des figures d'attaque triangulaires sur la plupart des cristaux $(80 \%$ d'entre eux sont orientées (111)). La figure 1 montre un aspect typique de la surface des diodes étudiées.

Les densitēs de dislocations $N_{d i s}$ sont évaluées en moyennant la rêpartition des figures d'attaque. Seules les dislocations qui émergent ã la surface des grains sont ainsi comptées, ce qui minore considérablement leur densité réelle. Ma is les anaiyses par topographie $X$ (difficilement exploitables pour le comptage à cause des valeurs généralement élevées de $N_{\text {dis }}$ ) confirment les évolutions des densités de dislocations obtenues à partir des figures d'attaque. La concentration en carbone est déterminée par absorption infrarouge différentiel1e sur les emplacements des diodes mesas.

\section{III - RESULTATS EXPERIMENTAUX}

La figure 2 représente la variation de la longueur de diffusion des électrons $L_{n}$ avec la longueur totale de joints de grains par unité de surface $L j$ dans des diodes du lingot 121. Le graphe montre bien l'influence des joints de grains, défaut majeur dans le silicium polycristallin. 




(a)

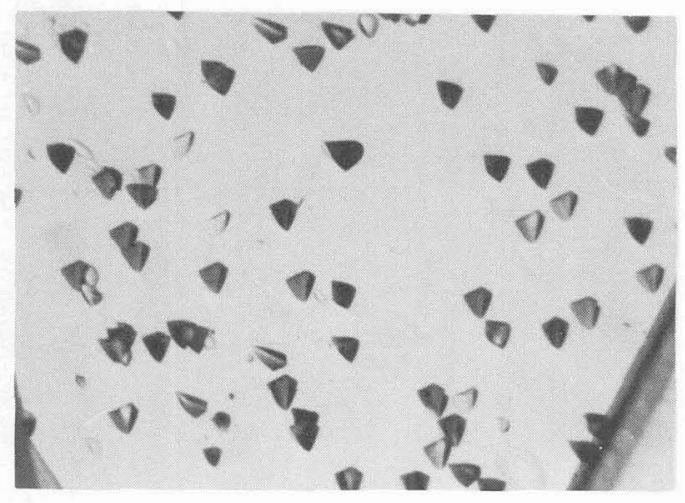

(b)

Fig.1. (a) Défauts typiques d'une diode mesa peu disloquée $(G=40)$

(b) Aggrandissement d'une région très disloquée montrant les figures $d^{\prime}$ attaque $(G=400)$.

(a) Typical defects of a mesa diode containing few dislocations $(G=40$ )

(b) Magnification of a heavily dislocated region of a mesa diode showing the triangular etch pits $(G=400)$



Fig.2. Variation de la longueur de diffusion avec $L_{j}$.

Dependence of diffusion length on total length of grain boundaries per unit area $L_{j}$. 
La dispersion des résultats de la figure 2 peut s'expliquer en grande partie par la contribution aux mécanismes de recombinaison des dislocations, dont la densité moyenne varie d'une diode à l'autre. En effet en ne retenant que les diodes des lingots 121 et 123 dans lesquelles les valeurs de $L_{j}$ sont approximativement les mêmes (20< $L_{j}<30 \mathrm{~cm} / \mathrm{cm}^{2}$ ce qui correspond à des dimensions de grains de l'ordre de 500um) on constate que $L_{n}$ diminue quand $N_{\text {dis }}$ dépasse $10^{3} \mathrm{~cm}^{-2}$ comme Te montre la figure 3 .

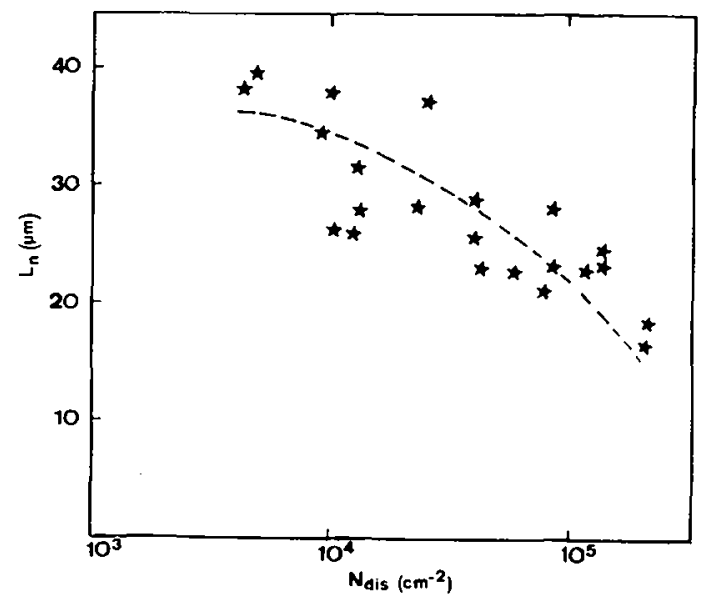

Fig.3. Variation de la longueur de diffusion avec la densité de dislocations pour des diodes présentant approximativement le même $L_{j}$.

Variation of diffusion length with dislocation density for diodes presenting approximately the same $L_{j}$ value.

Les lois de variation de $L_{n}$ avec $L_{j}$ et de $L_{n}$ avec $H_{d i s}$ peuvent s'obtenir sêparemment en représentant $L_{n}^{-1}$ en fonction de $L_{j}$ pour des diodes présentant des densités de dislocations voisines et $L_{n}^{-2}$ en fonction de $N_{\text {dis }}$ pour des diodes présentant approximativement la même valeur de $L_{j}$.

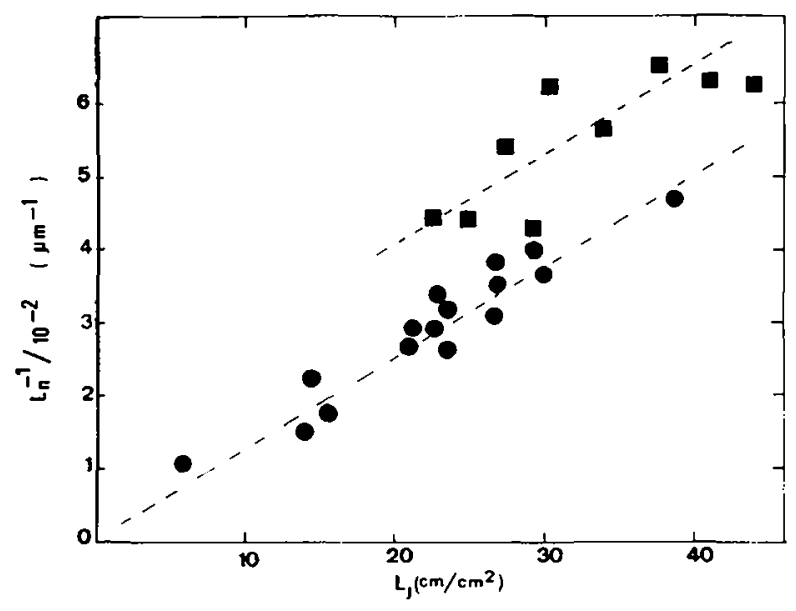

Fig.4. Variation de $L_{n}^{-1}$ avec $L_{j}$, pour des diodes présentant approximativement la même densité de dislocations

- $\mathrm{N}_{\text {dis }}>10^{5} \mathrm{~cm}^{-2}$
- $\mathrm{N}_{\text {dis }}<2.10^{4} \mathrm{~cm}^{-2}$

variation of $L_{n}^{-1}$ versus $L_{j}$ for diodes presenting approximately the same density of dislocations. 


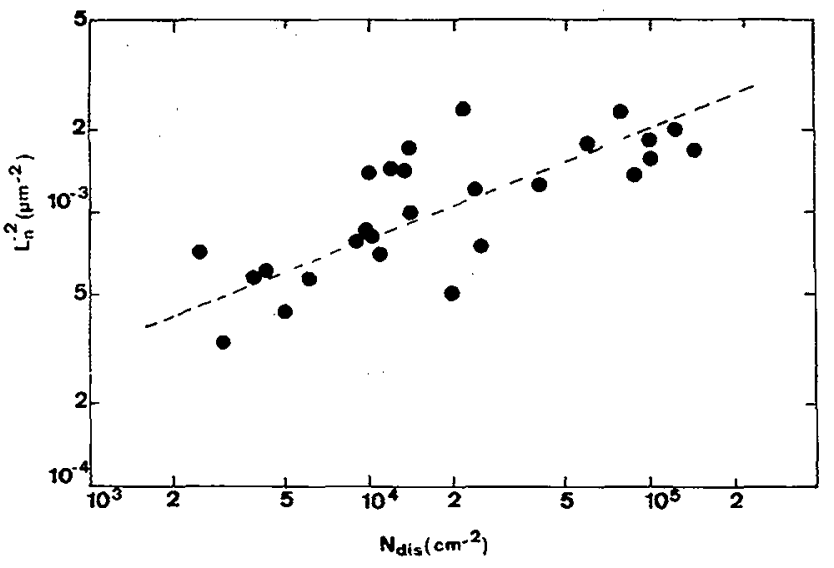

Fig.5. Variation de $L_{n}^{-2}$ avec $N_{\text {dis }}$ pour des diodes présentant approximativement Te même $L_{j}$.

Variation of $L_{n}^{-2}$ versus $H_{d i s}$ for diodes presenting approximately the same $L_{j}$ value.

Les figures 4 et 5 montrent que

$$
L_{n}^{-1} \simeq A L_{j}+B \quad \text { et } \quad L_{n}^{-2} \simeq C\left(N_{d i s}\right)^{1 / 4}+D \text {. }
$$

Aussi pour le silicium polycristallin la règle de Macthiessen s'écrirait, puisque $L_{n}=\sqrt{D_{n} \tau_{n}}$ :

$$
\frac{1}{L_{n}^{2}}=a L_{j}^{2}+b\left(N_{d i s}\right)^{1 / 4}+c \text { (impuretēs) }+\ldots
$$

La dispersion des résuitats de la figure 5 est encore liée au difficile comptage des dislocations et T'exposant $1 / 4$ ne peut être donné qu'à titre indicatif. Cet exposant est petit si on le compare à celui trouvé par INOUE et al. dans des siliciums polycristallins Hacker et Monsanto $/ 7 /\left(L_{n}^{-2} \div N_{d i s}\right)$. Hais ces auteurs $n$ 'ont pris en compte que les dislocations actives décelées par E.B.I.C. Par ailleurs, GLAENZER et JORDAN /2/ ont montré que dans du silicium monocristallin contenant peu d'oxygène, les dislocations créées par déformation plastique ne contribuent pas aux mécanismes de recombinaison. Aussi la variation de $L_{n}$ avec $N_{\text {dis }}$ que nous avons trouvée dans le silicium polycristallin CGE préparē sous vide, pourrait s'expliquer par une faible proportion de dislocations actives.

La variation du photocourant $I_{C C}$ avec $L_{j}$ est très comparable à celle de $L_{n}$. La figure 6 montre que $I_{C c}$ varie, comme $L_{n}$ avec la densité de dislocations, quand

$20<L_{j}<30 \mathrm{~cm} / \mathrm{cm}^{2}$. La variation de la phototension $V_{c o}$ avec $N_{\text {dis }}$ est plus accentuée que celle de $I_{C c}$, car les dislocations agissent sur $V_{c o}$ de deux façons : par leur contribution aux mécanismes de recombinaison et par l'augmentation des courants de fuite des diodes.

Les longueurs de diffusion varient aussi avec la concentration en carbone comme le montre la figure 7 où sonî représentés les résultats relatifis aux diodes mesa provenant des lingots 121 et 123 . Le carbone ne devrait pas influencer directement les durées de vie /4/ (donc les longueurs de diffusion) dans le silicium polycristallin. Toutefois, les concentrations en carbone mesurées, approchent ou dépassent la limite de solubilité qui varie selon les auteurs de $3,5.1017$ à $5.10^{17} \mathrm{~cm}^{-3} / 4,8 /$. De telles concentrations peuvent engendrer des microdéfauts $14,10 /$, réduire la taille des grains et creer des dislocations $15,9,10,11$. Le carbone pourrait ainsi agir par j'intermëdiaire de $L_{j}$ et de $N_{d i s}$. 
C'est aussi ce que suggèrent nos rësultats. En effet, la taille moyenne des grains du Tingot $123(500 \mu \mathrm{m})$ est sensiblement plus grande que celle des grains du lingot 121 sursaturé en carbone $(400 \mu \mathrm{m})$ et la figure 8 montre que la densité moyenne de dislocation dans une diode mesa dépend de la concentration en carbone [C].

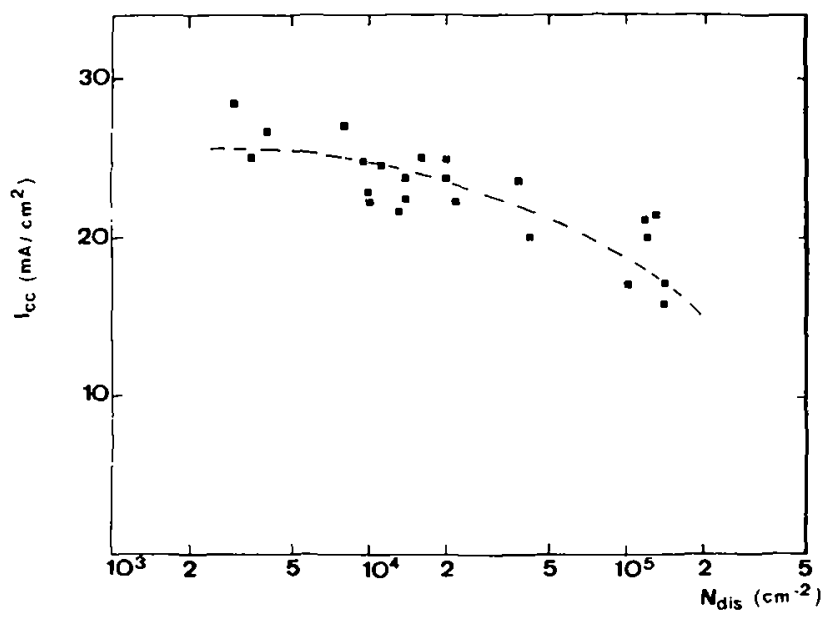

Fig.6. Variation du photocourant de court-circuit avec la densité de dislocations pour des diodes dont les valeurs de $\mathrm{L}_{j}$ sont voisines.

Variation of the short circuit photocurrent versus the dislocation density for diodes which the $L_{j}$ values are quite similar.

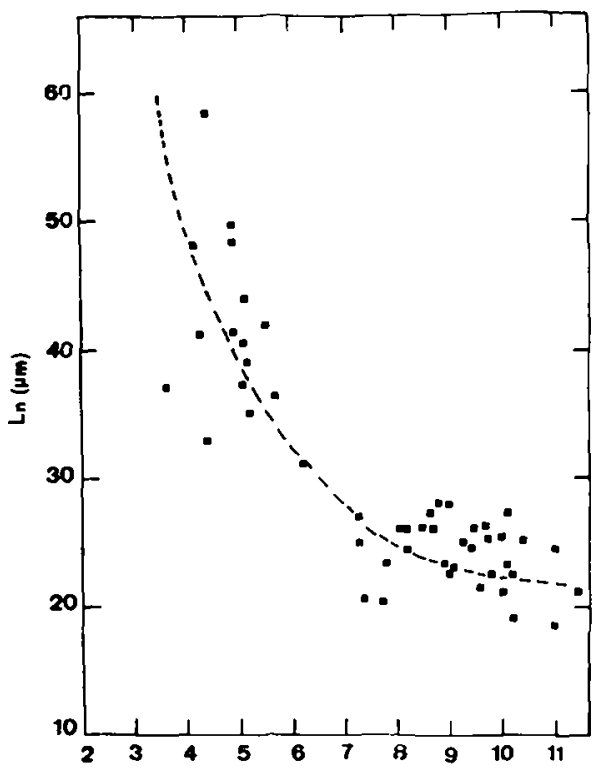

Fig.7. Variation de la longueur de diffusion avec la concentration en carbone dans les diodes mesa.

Variation of diffusion length versus carbon concentration in the mesa diodes.

[C] $\left(10^{7} \mathrm{~cm}^{-3}\right)$ 


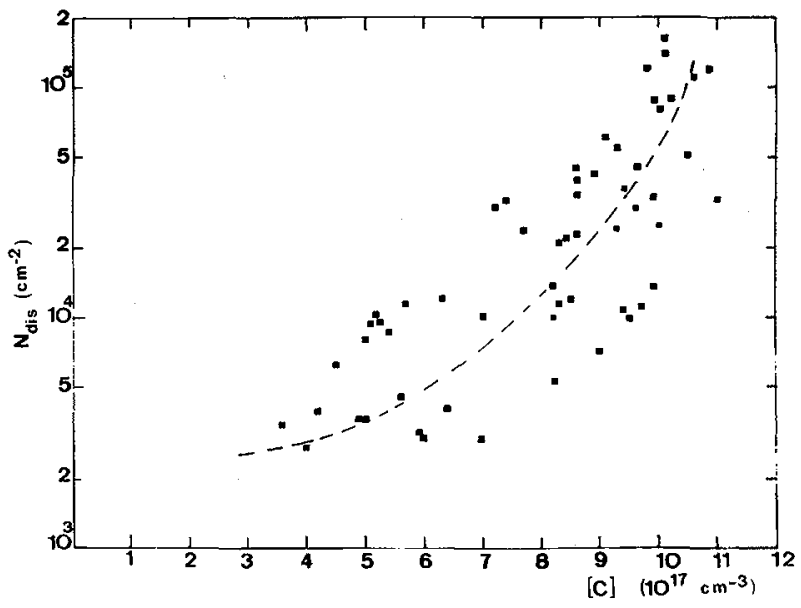

Fig.8. Variation de la densité de dislocations en fonction de la concentration en carbone dans les diodes mesa.

Variation of dislocation density versus carbon concentration in the mesa diodes.

Malgrẽ 1 a dispersion des valeurs expérimentales (dues en grande partie aux difficultés de comptage des figures d'attaque), on observe une nette augmentation de $N_{\text {dis }}$ quand $[C]$ dẻpasse $5.10^{17} \mathrm{~cm}^{-3}$. Pour des valeurs de [C] inférieures à $3.10^{17} \mathrm{~cm}^{-3}$, $N$ semble tendre vers une limite infërieure de 1 ordre de $10^{3} \mathrm{~cm}^{-2}$ qui résulterait dés dislocations créées par les contraintes développées au cours du refroidissement rapide des lingots, ou des traitements thermiques nécessaires à la fabrication des jonctions $N+P$.

\section{IV - CONCLUSION}

Dans le silicium polycristallin C.G.E. les joints de grains constituent le défaut cristallographique majeur. Les longueurs de diffusion varient en effet comme l'inverse de leur longueur ramenée à l'unité de surface. Majs les dislocations sont également nocives dès que leur densité moyenne dépasse $10^{4} \mathrm{~cm}^{-2}$. Les longueurs de dífiusion varient proportionnellement à 1 'inverse de la racine huitième de la densité de dislocations. Une variation aussi modérée résulterait de la faible proportion de dislocations actives dans ce silicium préparé sous vide.

Une corrélation existe entre les densités de diṣlocations supérieures à $10^{3} \mathrm{~cm}^{-2}$ et les concentrations en carbone supérieures à $3.10^{17} \mathrm{~cm}^{-3}$. En créant de nombreuses dislocations et en limitant la taille des grains ces concentrations élevées en carbone peuvent dégrader les longueurs de diffusion.

Ce travait bénéricie de l'aide du CNRS-PIRSEH - APP n 1004/3003. et du COMES contrat $n^{\circ} 81.11 .013 .3413$.

Nous tenons à remercier les Laboratoires de Marcoussis C.G.E. (M. FALLY), et PHOTOWATT Int. S.A. (M. DONON) pour la fourniture des plaquettes de silicium et des photopiles. 


\section{$V$ - BIBLIOGRAPHIE}

1/ MATHIAN G., AMZIL H., ZEHAF M., CREST J.P., PSAILA E., MARTINUZZI S. and OUALID J., Solid State Electronics, 26, (1983) 131.

12/ GLAENZER R.H. et JORDAN A.G., Solid State Electronics, 12, (1969) 247.

13/ SCHROTER W. and LABUSCH R., Phys.Stat.So1., 36 (1969), 359.

14/ PIZZINi S., Solar Energy Materials, 6, (1982), 253.

15/ KOBELSEN B.0., MUHLBAUER A., Solid State Electronics, 25, (1982), 759.

16/ FALLY J. and GUENEL C., Fourth E.C. Photovoltaîc Solar Energy Conf., Stresa, (1982), 955.

17/ INOUE N., WILSEN C.W. and JONES K.A., Solar Cel1s, 3, (1981), 35.

/8/ BEAN A.R. and NEWMAN R.C., J.Phys.Chem.Solids, 32, (1971), 1211.

19/ KISHINO S., MATSUSHITA Y: and KANAMORI M., App1.Phys.Lett., 35 (1979), 213.

110/ WOLTMER F.W. and PADOVANI F.A., Semiconductor silicon (Edited by HUFF H.R. and BURGESS R.R.). The Electrochemical Soc. Princeton, New Jersey (1973) 75.

11/ HENDRIKS M., RADELAAR S., de KEIJSER Th.H. and DELNEZ R., J. de Phys. 43, (1982), C1.307. 